\title{
A comparison of minimally invasive and standard aortic valve replacement
}

Jarosław Stoliński, MD, PhD, ${ }^{\text {a }}$ Dariusz Plicner, $\mathrm{MD}, \mathrm{PhD},{ }^{\mathrm{a}}$ Grzegorz Grudzień, $\mathrm{MD}, \mathrm{PhD},{ }^{\mathrm{a}}$

Marcin Wasowicz, MD, PhD, ${ }^{b}$ Robert Musiał, MD, ${ }^{c}$ Janusz Andres, MD, PhD, ${ }^{c}$ and

Bogusław Kapelak, MD, $\mathrm{PhD}^{\mathrm{a}}$

\section{ABSTRACT}

Objective: The study objective was to compare aortic valve replacement through a right anterior minithoracotomy with aortic valve replacement through a median sternotomy.

Methods: With propensity score matching, we selected 211 patients after aortic valve replacement through a right anterior minithoracotomy and 211 patients after aortic valve replacement who underwent operation between January 2010 and December 2013. Perioperative outcomes were analyzed, and multivariable logistic regression analysis of risk factors of postoperative morbidity was performed.

Results: For propensity score-matched patients, hospital mortality was $1.0 \%$ in the aortic valve replacement through a right anterior minithoracotomy group and $1.4 \%$ in the aortic valve replacement group $(P=1.000)$. Stroke occurred in $0.5 \%$ versus $1.4 \%(P=.615)$, myocardial infarction occurred in $1.4 \%$ versus $1.9 \%$ $(P=1.000)$, and new onset of atrial fibrillation occurred in $12.8 \%$ versus $24.2 \%(P=.003)$ of patients in the aortic valve replacement through a right anterior minithoracotomy and aortic valve replacement groups, respectively. Postoperative drainage was $353.5 \pm 248.6 \mathrm{~mL}$ versus $544.3 \pm 324.5 \mathrm{~mL}(P<.001)$ and blood transfusion was required for $48.8 \%$ versus $67.3 \%(P<.001)$ of patients in the aortic valve replacement through a right anterior minithoracotomy and aortic valve replacement groups, respectively. Mediastinitis occurred in $2.8 \%$ of patients after aortic valve replacement and in $0.0 \%$ of patients after aortic valve replacement through a right anterior minithoracotomy surgery $(P=.040)$. Intensive care unit stay $(1.3 \pm 1.2$ days vs $2.6 \pm 2.6$ days $)$ and hospital stay $(5.7 \pm 1.6$ days vs $8.7 \pm 4.4$ days) were statistically significantly shorter in the aortic valve replacement through a right anterior minithoracotomy group. Aortic valve replacement through a right anterior minithoracotomy surgery resulted in reduced postoperative morbidity (odds ratio, $0.4 ; P<.001$ ) and postoperative bleeding and blood transfusion requirements (odds ratio, $0.4 ; P<.001$ ).

Conclusions: Aortic valve replacement through a right anterior minithoracotomy surgery resulted in a reduced infection rate, diminished postoperative bleeding and blood transfusion requirements, reduced occurrence of new onset of atrial fibrillation, and shorter intensive care unit and hospital stays. (J Thorac Cardiovasc Surg 2016;152:1030-9)

From the Departments of ${ }^{a}$ Cardiovascular Surgery and Transplantology and ${ }^{\mathrm{c}}$ Anesthesiology and Intensive Therapy, Jagiellonian University in Cracow, John Paul II Hospital, Cracow, Poland; ${ }^{b}$ Department of Anesthesia and Pain Management, Toronto General Hospital, Department of Anesthesia, Faculty of Medicine, University of Toronto, Toronto, Ontario, Canada.

M.W. is supported by a Merit Award from the Department of Anesthesia, University of Toronto.

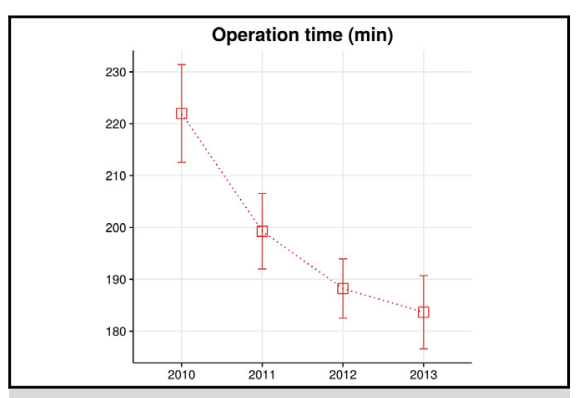

Operation time indicating a learning curve for RT-AVR surgery.

\section{Central Message}

RT-AVR surgery results in a reduced complication rate compared with conventional AVR surgery via a median sternotomy.

\section{Perspective}

RT-AVR surgery appeared to be feasible, safe, and efficient. Postoperative morbidity was diminished, and the mortality rate was comparable to that of conventional AVR surgery. Minimally invasive AVR surgery should be considered when isolated surgical AVR is indicated, keeping in mind major limitations such as left-sided aortas and right pleural adhesions.

See Editorial Commentary page 1040.
Received for publication Dec 15, 2015; revisions received May 20, 2016; accepted for publication June 12, 2016; available ahead of print July 20, 2016.

Address for reprints: Jarosław Stoliński, MD, PhD, Department of Cardiovascular Surgery and Transplantology, Jagiellonian University in Cracow, John Paul II Hospital, Prądnicka St 80, 31-202 Cracow, Poland (E-mail: jstolinski@gmail.com). 0022-5223/\$36.00

Copyright (C) 2016 by The American Association for Thoracic Surgery http://dx.doi.org/10.1016/j.jtcvs.2016.06.012 


\section{Abbreviations and Acronyms \\ AVA $=$ aortic valve area \\ AVR $=$ aortic valve replacement \\ CPB $=$ cardiopulmonary bypass \\ $\mathrm{EF} \quad=$ ejection fraction \\ EuroSCORE $=$ European System for Cardiac \\ Operative Risk Evaluation \\ ICU $=$ intensive care unit \\ LCOS = low cardiac output syndrome \\ MDCT = multidetector computed tomography \\ PSM $=$ propensity score matching \\ RT-AVR = aortic valve replacement through a right anterior minithoracotomy}

Scanning this QR code will take you to a procedural video.

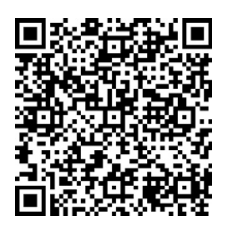

At the end of the last century, a conventional open procedure using a median sternotomy was the technique of choice in most cardiac surgical centers. ${ }^{1}$ Less-invasive surgical treatment of patients with valvular heart disease entered clinical practice in 1996, and since then, it has started to gain worldwide acceptance., ${ }^{2,3}$

Publications describing the safety, efficacy, mortality, and morbidity associated with the minimally invasive right anterior minithoracotomy approach in aortic valve surgery are still relatively scarce in medical literature. ${ }^{4}$ Currently, minimally invasive aortic valve surgery becomes an option when surgical aortic valve replacement (AVR) is indicated. ${ }^{5}$

The aim of this study was to answer the question if the perioperative outcomes of patients after minimally invasive aortic valve replacement through a right anterior minithoracotomy (RT-AVR) can be improved compared with patients after a conventional median sternotomy (AVR).

\section{MATERIALS AND METHODS Study Design}

This was an observational cohort study performed in accordance with the Declaration of Helsinki and the consensus guidelines expressed by the Strengthening the Reporting of Observational Studies in Epidemiology statement. $^{6}$ Approval from the Institutional Ethical Review Board (Regional Medical Chamber, October 10, 2010) was obtained, and written informed consent was obtained from all patients. The analysis is based on prospectively gathered preoperative data and in-hospital outcomes of 221 patients who underwent RT-AVR surgery between January 2010 and December 2013. The control group consisted of 316 patients who underwent operation during the same period, for whom standard AVR surgery was performed.
Exclusion criteria were emergency operations, including infective endocarditis, previous cardiac surgical procedures, and left ventricle ejection fraction (EF) less than $30 \%$. It was the policy of our department that these higher-risk patients undergo operation through a conventional median sternotomy approach. We applied propensity score matching (PSM) to alleviate differences in preoperative patient characteristics and to create 2 comparable study groups.

The decision to perform RT-AVR was based on preoperative multidetector computed tomography (MDCT) examination applying the methodology described by Glauber and colleagues. ${ }^{8}$ Contraindications for RT-AVR surgery were difficult access to the aorta, when the aorta was positioned more to the left side of the chest, a history of right-sided pleural effusion, thorax trauma with rib fracture, and other thorax deformities, all which can suggest the presence of pleural adhesions. ${ }^{8}$

The time of discharge from the intensive care unit (ICU) or hospital was determined by medical judgment and patient threshold for discharge. ${ }^{9}$ On the basis of PSM patients, a multivariable logistic regression analysis describing risk factors of postoperative morbidity, bleeding, and blood transfusion requirements was conducted. Because of the low number of particular complications, a dependent variable analyzed in the model was postoperative complications in total. Preoperative comorbidities, patient demographics, surgical technique, hemoglobin concentration 1 day before surgery and at ICU arrival, and length of cardiopulmonary bypass (CPB) were included as independent variables in the model.

\section{Definitions of Perioperative Events}

For hospital mortality, we defined death as occurring within 1 month after surgery or during the same hospitalization period if the hospitalization was prolonged more than 1 month. ${ }^{10}$ An online calculator (http://www. euroscore.org/calc.html) was used to calculate and predict hospital mortality based on the European System for Cardiac Operative Risk Evaluation (EuroSCORE) II system. ${ }^{10}$

A stroke was defined as new central neurologic deficit persisting more than 72 hours. $^{11}$ We recognized perioperative myocardial infarction when ST-segment changes on serial electrocardiograms were observed and troponin I level 24 hours after surgery was at least $1.0 \mathrm{ng} / \mathrm{mL} .{ }^{11}$ Preoperative renal insufficiency was defined as baseline serum creatinine level greater than $2.0 \mathrm{mg} / \mathrm{dL} .^{12}$ According to our protocol, blood transfusion was required when the hemoglobin level was less than $8 \mathrm{~g} / \mathrm{dL}$.

\section{Surgical Technique}

For preoperative planning of the RT-AVR surgery, patients underwent imaging using MDCT (Siemens Healthcare, Erlangen, Germany). ${ }^{8}$ For RT-AVR surgery, tracheal intubation was performed with a double-lumen tracheal tube. Vacuum-assisted CPB was instituted by peripheral cannulation of the femoral vessels through a 2- to 3-cm skin incision just below the level of the femoral ligament. The femoral artery usually was cannulated with a $19 \mathrm{~F}$ or $21 \mathrm{~F}$ arterial cannula (Bio-Medicus; Medtronic, Minneapolis, Minn), and the femoral vein was cannulated with a $25 \mathrm{~F}$ venous cannula (Quick Draw, Edwards Lifesciences, Irvine, Calif). Both cannulas were placed using the Seldinger technique under transesophageal echocardiography control. In rare cases when significant peripheral vascular disease was present or femoral vessels were small in diameter, central ascending aortic cannulation was performed with the same arterial cannula.

A 5- to 6-cm transverse incision usually was made over the right third rib, and the chest was entered through the second or third intercostal space on the right side (Video 1). The third costal cartilage usually was transected to avoid rib fracture. At the completion of the operation, the rib was reattached to the sternum. The right internal thoracic vessels were transected and secured. $\mathrm{CO}_{2}$ insufflation into the right hemithorax was performed with a flow of $5 \mathrm{~L} / \mathrm{min}$. The left ventricle vent was placed through the right superior pulmonary vein, an aortic crossclamp was applied through the incision, and antegrade warm blood cardioplegia was delivered through a 


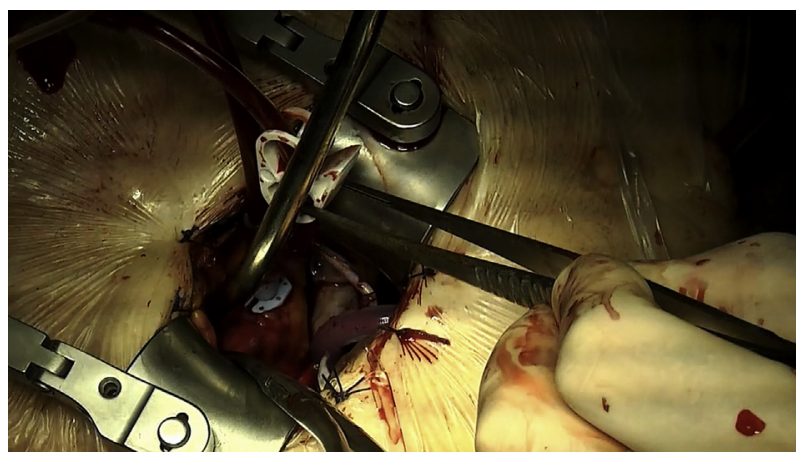

VIDEO 1. Aortic valve replacement through a right anterior minithoracotomy. Video available at http://www.jtcvsonline.org/article/S0022-5223(16) 30570-0/addons.

catheter placed in the ascending aorta with repeated doses given directly to the coronary ostia $\left(36^{\circ} \mathrm{C}\right.$, first a dose with potassium concentration of $20 \mathrm{mmol} / \mathrm{L}$ with a flow of $300 \mathrm{~mL} / \mathrm{min}$ for 2 minutes and every 20 minutes repeated doses with potassium concentration of $10 \mathrm{mmol} / \mathrm{L}$ with a flow of $200 \mathrm{~mL} / \mathrm{min}$ for 2 minutes). Thoracoscopic visualization was helpful in selected cases, when the aortic valve was positioned deep with a long distance between the chest wall and the level of the aortic annulus. ${ }^{4,5}$

Conventional AVR surgery was performed through the full-length median sternotomy, $\mathrm{CO}_{2}$ insufflation was used, antegrade blood warm cardioplegia was applied, and aortic valve surgery was performed using standard techniques. The same surgeons were performing both operations.

\section{Statistical Analysis}

PSM analysis was performed using multiple logistic regression analysis. Input model for PSM included the following clinical preoperative variables: female sex, age, AVA, bicuspid aortic valve, body mass index, aortic stenosis, EuroSCORE II, renal insufficiency, hemodialysis, diabetes, chronic obstructive pulmonary disease, EF, hypertension, peripheral vascular disease, coronary artery disease, cerebrovascular disease, New York Heart Association class III or IV, history of myocardial infarction, history of atrial fibrillation, and hemoglobin concentration ( 1 day before surgery). The final model was obtained by stepwise regression using a backward elimination approach in which independent variables with $P$ values greater than .05 are sequentially removed until only statistically significant variables remain. Those were female sex + AVA + EF + history of atrial fibrillation + hemoglobin concentration (1 day before surgery).

PSM was performed using the MatchIt package in R 3.0. ${ }^{13}$ The model was used in PSM using the nearest neighbor method. To decrease selection bias, a caliper equal to 0.25 standard deviations of logit distance measure (propensity score) was used. Quality of the PSM was assessed using the area under the receiver operating characteristic curve and Hosmer-Lemeshow goodness of fit test.

In the PSM population, categoric variables were expressed as counts and percentages. Empirical distribution of continuous variables was described using mean and standard deviation, and additionally described using median and quartiles. For continuous variables, statistical significance of differences between 2 independent groups was assessed using the Mann-Whitney test. For 2 categoric variables, the Fisher exact test or chi-square test was used. Poisson regression analysis for the length of ICU stay and hospital stay was performed and reported using estimate and standard error. Associations between a binary dependent variable and multiple independent variables were assessed by the use of a bias-reduced logistic regression model and reported using odds ratios (ORs) with $95 \%$ confidence intervals (CIs). All statistical analyses were performed using R 3.0. ${ }^{13}$

\section{RESULTS}

After MDCT scans, we preoperatively excluded 12 patients from RT-AVR surgery. For 9 patients, the reason was the anatomic position of the ascending aorta located deep under the sternum. ${ }^{8}$ For these patients, conventional AVR surgery was performed. An additional 3 patients qualified for transfemoral aortic valve implantation because of ascending aorta calcifications.

During the study period, there were 221 patients after RT-AVR surgery and 316 patients after AVR surgery who met exclusion criteria. After PSM, 211 patients in the RT-AVR group and 211 patients in the AVR group were included in the study. The final model for PSM included female sex + AVA + EF + history of atrial fibrillation + hemoglobin concentration (1 day before surgery) (Table 1 ).

The quality of the propensity score model was assessed. Area under the receiver operating characteristic curve (receiver operating characteristic) showing the effectiveness of a patient's qualification to the study group was 0.6808 (Hosmer-Lemeshow goodness of fit test: $P$ value $=.904)$, which indicated that there was no evidence of model incorrectness. Standardized differences between RT-AVR and AVR groups for each baseline variable included in the propensity score model are presented in Table 2.

After PSM, both study groups were similar in patients' demographics and preoperative clinical characteristics (Table 2). Conversion to a median sternotomy was

TABLE 1. Final model for propensity score matching

\begin{tabular}{|c|c|c|c|c|c|c|}
\hline & Estimate & Standard error & $Z$ value & OR & $95 \%$ CI & $P$ value \\
\hline (Intercept) & -3.802 & 0.622 & -6.109 & 0.022 & $0.0-0.1$ & .000 \\
\hline Female sex & -0.715 & 0.203 & -3.530 & 0.489 & $0.3-0.7$ & .000 \\
\hline AVA & 0.993 & 0.317 & 3.129 & 2.700 & $1.5-5.1$ & .002 \\
\hline Left ventricle EF & 0.038 & 0.007 & 5.542 & 1.039 & $1.0-1.1$ & .000 \\
\hline History of atrial fibrillation & 0.647 & 0.299 & 2.162 & 1.910 & $1.1-3.5$ & .031 \\
\hline $\mathrm{Hb}$ concentration ( $1 \mathrm{~d}$ before surgery) & 0.095 & 0.033 & 2.914 & 1.099 & $1.0-1.2$ & .004 \\
\hline
\end{tabular}

The final model was obtained by stepwise regression using backward elimination approach in which independent variables with $P$ values greater than .05 are sequentially removed until only statistically significant variables remain. Those were female sex + AVA + EF + history of atrial fibrillation $+\mathrm{Hb}$ ( 1 day before surgery). This model was used in PSM using nearest neighbor method. To decrease selection bias, a caliper equal to 0.25 was used. AVA measured with Doppler echocardiography. OR, Odds ratio; $C I$, confidence interval; $A V A$, aortic valve area; $E F$, ejection fraction; $H b$, hemoglobin. 
TABLE 2. Preoperative characteristics of patients

\begin{tabular}{|c|c|c|c|c|}
\hline \multicolumn{5}{|c|}{ Unmatched patients } \\
\hline Characteristics & $\begin{array}{c}\text { RT-AVR group } \\
\mathbf{n}=\mathbf{2 2 1}\end{array}$ & $\begin{array}{c}\text { AVR group } \\
\mathrm{n}=316\end{array}$ & $P$ value & Standardized differences \\
\hline Female, \% (n) & $42.1(93)$ & $51.0(161)$ & .043 & -0.177 \\
\hline Age, $y \pm S D$ & $\begin{array}{c}67.9 \pm 10.7 \\
69.0[63.0 ; 75.0]\end{array}$ & $\begin{array}{c}68.3 \pm 11.7 \\
71.0[62.0 ; 76.0]\end{array}$ & .683 & -0.036 \\
\hline Age $>80$ y, $\%(n)$ & $5.0(11)$ & $10.1(32)$ & .030 & -0.194 \\
\hline BMI, $\mathrm{kg} / \mathrm{m}^{2} \pm \mathrm{SD}$ & $\begin{array}{c}26.9 \pm 5.3 \\
26.6[23.5 ; 29.6]\end{array}$ & $\begin{array}{c}27.9 \pm 7.6 \\
26.3[22.5 ; 35.5]\end{array}$ & .070 & -0.164 \\
\hline Aortic stenosis, \% (n) & $71.5(158)$ & $70.9(224)$ & .878 & 0.013 \\
\hline $\mathrm{AVA}, \mathrm{cm}^{2} \pm \mathrm{SD}$ & $\begin{array}{c}0.7 \pm 0.3 \\
0.7[0.5 ; 1.0]\end{array}$ & $\begin{array}{c}0.7 \pm 0.3 \\
0.6[0.5 ; 0.9]\end{array}$ & .227 & 0.141 \\
\hline $\mathrm{BAV}, \%(\mathrm{n})$ & $31.2(69)$ & $33.5(106)$ & .637 & -0.050 \\
\hline Hemodialysis, \% (n) & $0.5(1)$ & $0.6(2)$ & .782 & -0.025 \\
\hline Diabetes, \% (n) & $13.1(29)$ & $17.7(56)$ & .150 & -0.127 \\
\hline COPD, \% (n) & $7.2(16)$ & $8.2(26)$ & .675 & -0.037 \\
\hline $\mathrm{EF} \% \pm \mathrm{SD}$ & $\begin{array}{c}48.8 \pm 12.2 \\
50.0[40.0 ; 60.0]\end{array}$ & $\begin{array}{c}42.5 \pm 15.0 \\
40.0[35.0 ; 55.0]\end{array}$ & $<.001$ & 0.458 \\
\hline $\mathrm{EF}>40 \%, \%(\mathrm{n})$ & $77.4(171)$ & $55.1(174)$ & $<.001$ & 0.459 \\
\hline Hypertension, \% (n) & $45.7(101)$ & $47.8(151)$ & .634 & -0.042 \\
\hline $\mathrm{Hb}$ concentration ( $1 \mathrm{~d}$ before surgery), $\mathrm{g} / \mathrm{dL} \pm \mathrm{SD}$ & $13.7 \pm 2.8$ & $12.9 \pm 3.4$ & .010 & 0.258 \\
\hline Peripheral vascular disease, $\%(n)$ & $13.6(30)$ & $21.8(69)$ & .015 & -0.215 \\
\hline Cerebrovascular disease, $\%(n)$ & $10.9(24)$ & $11.1(35)$ & .937 & -0.007 \\
\hline Coronary artery disease, $\%(n)$ & $13.1(29)$ & $16.8(53)$ & .247 & -0.081 \\
\hline NYHA class III or IV, \% (n) & $25.5(57)$ & $28.2(89)$ & .543 & -0.053 \\
\hline History of myocardial infarction, $\%$ (n) & $11.3(25)$ & $13.9(44)$ & .373 & -0.078 \\
\hline History of atrial fibrillation, $\%$ (n) & $13.1(29)$ & $9.5(30)$ & .237 & 0.114 \\
\hline Predicted mortality, EuroSCORE II $\% \pm$ SD & $\begin{array}{c}3.2 \pm 1.1 \\
3.1[2.7 ; 3.5]\end{array}$ & $\begin{array}{c}3.4 \pm 1.9 \\
3.0[2.7 ; 3.5]\end{array}$ & .066 & -0.182 \\
\hline
\end{tabular}

Patients after propensity score matching

\begin{tabular}{|c|c|c|c|c|}
\hline Characteristics & $\begin{array}{c}\text { RT-AVR group } \\
n=211\end{array}$ & $\begin{array}{c}\text { AVR group } \\
n=211\end{array}$ & $P$ value & Standardized differences \\
\hline Female, \% (n) & $43.6(92)$ & $42.2(89)$ & .844 & 0.029 \\
\hline Age, $y \pm S D$ & $\begin{array}{c}68.0 \pm 10.6 \\
69.0[63.0 ; 76.0]\end{array}$ & $\begin{array}{c}68.1 \pm 11.8 \\
71.0[62.0 ; 76.0]\end{array}$ & .448 & -0.006 \\
\hline Age $>80$ y, \% (n) & $5.2(11)$ & $8.5(18)$ & .248 & -0.131 \\
\hline BMI, $\mathrm{kg} / \mathrm{m}^{2} \pm \mathrm{SD}$ & $\begin{array}{c}27.0 \pm 5.3 \\
26.6[23.6 ; 29.6]\end{array}$ & $\begin{array}{c}27.7 \pm 7.4 \\
26.6[22.5 ; 35.5]\end{array}$ & .452 & -0.115 \\
\hline Aortic stenosis, \% (n) & $72.0(152)$ & $72.5(153)$ & 1.000 & -0.011 \\
\hline $\mathrm{AVA}, \mathrm{cm}^{2} \pm \mathrm{SD}$ & $\begin{array}{c}0.7 \pm 0.3 \\
0.6[0.5 ; 0.9]\end{array}$ & $\begin{array}{c}0.7 \pm 0.3 \\
0.7[0.5 ; 0.9]\end{array}$ & .898 & 0.067 \\
\hline $\mathrm{BAV}, \%(\mathrm{n})$ & $31.2(66)$ & $34.1(72)$ & .604 & -0.061 \\
\hline Renal insufficiency, \% (n) & $9.5(20)$ & $9.0(19)$ & 1.000 & 0.016 \\
\hline Hemodialysis, \% (n) & $0.5(1)$ & $0.5(1)$ & 1.000 & 0.000 \\
\hline Diabetes, \% (n) & $13.7(29)$ & $18.0(38)$ & .287 & -0.117 \\
\hline COPD, $\%(n)$ & $7.6(16)$ & $8.1(17)$ & 1.000 & -0.018 \\
\hline $\mathrm{EF} \% \pm \mathrm{SD}$ & $\begin{array}{c}48.3 \pm 12.2 \\
50.0[40.0 ; 60.0]\end{array}$ & $\begin{array}{c}47.0 \pm 15.4 \\
45.0[35.0 ; 60.0]\end{array}$ & .202 & 0.099 \\
\hline $\mathrm{EF}>40 \%, \%(n)$ & $76.3(161)$ & $67.7(143)$ & .065 & 0.189 \\
\hline Hypertension, \% (n) & $46.4(98)$ & $44.5(94)$ & .769 & 0.038 \\
\hline $\mathrm{Hb}$ concentration ( $1 \mathrm{~d}$ before surgery), $\mathrm{g} / \mathrm{dL} \pm \mathrm{SD}$ & $\begin{array}{c}13.6 \pm 2.6 \\
13.1[11.4 ; 16.2]\end{array}$ & $\begin{array}{c}13.4 \pm 3.0 \\
13.1[11.1 ; 16.0]\end{array}$ & .293 & 0.060 \\
\hline
\end{tabular}


TABLE 2. Continued

\begin{tabular}{lccrr}
\hline & Patients after propensity score matching & & \\
\multicolumn{1}{c}{ Characteristics } & RT-AVR group & AVR group & & \\
& $\mathbf{n}=\mathbf{2 1 1}$ & $\mathbf{n}=\mathbf{2 1 1}$ & $\boldsymbol{P}$ value & Standardized differences \\
\hline Peripheral vascular disease, \% (n) & $14.2(30)$ & $18.5(39)$ & .292 & -0.117 \\
Cerebrovascular disease, \% (n) & $10.9(23)$ & $9.0(19)$ & .626 & 0.063 \\
Coronary artery disease, \% (n) & $14.2(30)$ & $12.8(27)$ & .776 & 0.042 \\
NYHA class III or IV, \% (n) & $25.6(54)$ & $28.0(59)$ & .660 & -0.054 \\
History of myocardial infarction, \% (n) & $11.8(25)$ & $10.0(21)$ & .639 & 0.061 \\
History of atrial fibrillation, \% (n) & $12.3(26)$ & $11.4(24)$ & .880 & .184 \\
Predicted mortality, EuroSCORE II \% \pm SD & $3.2 \pm 1.1$ & $3.2 \pm 1.7$ & .029 \\
& $3.0[2.7 ; 3.5]$ & $2.9[2.5 ; 3.6]$ & & -0.016 \\
\hline
\end{tabular}

$\mathrm{n}=$ number of patients. Continuous variables are described by mean $\pm \mathrm{SD}$ and by median [first; third quartile]. Categoric variables are shown as a percentage. AVA measured with Doppler echocardiography. $R T$-AVR, Minimally invasive aortic valve replacement through a right anterior minithoracotomy; $A V R$, conventional aortic valve replacment through a median sternotomy; $S D$, standard deviation; $B M I$, body mass index; $A V A$, aortic valve area; $B A V$, bicuspid aortic valve; $C O P D$, chronic obstructive pulmonary disease; $E F$, ejection fraction; $\mathrm{Hb}$, hemoglobin concentration; NYHA, New York Heart Association; EuroSCORE, European System for Cardiac Operative Risk Evaluation.

necessary in 6 patients $(2.8 \%)$ in the RT-AVR group, and they were included and analyzed in the RT-AVR group with an intention-to-treat analysis. In 3 patients, conversion was necessary because of bleeding from the aortotomy line, which was difficult to control with small surgical access area. In 1 patient, conversion was performed after the pericardial sac was opened and the ascending aorta was located under the sternum, which was different than initially described by the MDCT report. In 2 patients, conversion to a median sternotomy was necessary after a right thoracotomy was made and dense pleural adhesions were found.

On the basis of the preoperative MDCT scans, 14 patients were disqualified from retrograde $\mathrm{CPB}$ perfusion. Disqualification was due to femoral artery calcification in 11 of these patients and an abdominal aortic aneurysm with thrombus in 3 of these patients. In those 14 patients, $\mathrm{CPB}$ with antegrade perfusion was performed.

Two patients $(1.0 \%)$ died in the RT-AVR group. Causes of death were as follows: massive stroke in 1 patient and perioperative myocardial infarction with cardiogenic shock in 1 patient, who had acute occlusion of the left anterior descending coronary artery that was treated with percutaneous coronary intervention. Three patients $(1.4 \%)$ died in the AVR group. Causes of death in this group were stroke in 1 patient and perioperative myocardial infarction with cardiogenic shock in 1 patient. In 1 patient, sudden cardiac arrest occurred on postoperative day 5; resuscitation was not successful, and autopsy did not reveal a cause of death.

Low cardiac output syndrome (LCOS) was diagnosed in 1 patient in the RT-AVR group and 1 patient in the AVR group. These patients had perioperative myocardial infarction, developed cardiogenic shock, and died. The other 2 patients in whom LCOS was diagnosed had low left ventricle EF before surgery.

Both hospital and ICU stays were shorter in the RT-AVR group. The aortic crossclamp, CPB, and entire operation times were longer in the RT-AVR group. Over time, differences disappeared between groups for the CPB and operation times. The aortic crossclamp time remained statistically significantly longer for patients in the RT-AVR group for the entire study period (Table 3).

Among patients in the RT-AVR group, there was a significant reduction in postoperative blood loss in the first 24 hours after surgery compared with the AVR group. Both the amount of blood transfused and the number of patients who required blood transfusion postoperatively were lower in the RT-AVR group. However, the incidence of reoperations for bleeding was not different between groups. In regard to postoperative complications, there was no difference between groups in terms of incidence of major organ dysfunction.

The incidence of new-onset atrial fibrillation was statistically significantly lower in the RT-AVR group in the postoperative period. Mediastinitis was not observed in the RT-AVR group and was diagnosed in $2.8 \%$ in the AVR group $(P=.040)$. Six patients $(2.8 \%)$ in the RT-AVR group had a healing complication within a groin wound, and 5 patients $(2.4 \%)$ had groin lymphocele. All of these wound-healing complications were successfully treated with surgical wound debridement and intravenous antibiotics.

There were no statistically significant differences between groups regarding pulmonary complications. Retained hemothoraces of the right hemithorax were removed thoracoscopically in 2 patients in the RT-AVR group during the postoperative period (Table 4).

There was a statistically significant reduction in CPB time, aortic crossclamp time, operation time, and postoperative drainage in the RT-AVR group during the study period. CPB time decreased from $133.0 \pm 28.2$ minutes in 2010 to $97.4 \pm 17.8$ minutes in $2013(P<.001)$. Aortic crossclamp time decreased from $90.9 \pm 20.3$ minutes in 2010 to $70.4 \pm 8.7$ minutes in $2013(P<.001)$. Operation time 
TABLE 3. Procedural characteristics and outcomes

\begin{tabular}{|c|c|c|c|}
\hline Characteristics & $\begin{array}{l}\text { RT-AVR group } \\
\quad \mathbf{n}=\mathbf{2 1 1} \\
\end{array}$ & $\begin{array}{c}\text { AVR group } \\
\mathbf{n}=\mathbf{2 1 1}\end{array}$ & $P$ value \\
\hline Perioperative mortality, $\%$ (n) & $1.0(2)$ & $1.4(3)$ & 1.000 \\
\hline Hospital stay, $\mathrm{d} \pm \mathrm{SD}$ & $\begin{array}{c}5.7 \pm 1.6 \\
5.0[5.0 ; 6.0]\end{array}$ & $\begin{array}{c}8.7 \pm 4.4 \\
7.0[5.0 ; 11.5]\end{array}$ & $<.001$ \\
\hline ICU stay, $\mathrm{d} \pm \mathrm{SD}$ & $\begin{array}{c}1.3 \pm 1.2 \\
1.0[1.0 ; 1.0]\end{array}$ & $\begin{array}{c}2.6 \pm 2.6 \\
2.0[1.0 ; 3.0]\end{array}$ & $<.001$ \\
\hline $\mathrm{CPB}$ with retrograde perfusion, $\%$ (n) & $96.2(203)$ & $0.0(0)$ & $<.001$ \\
\hline $\mathrm{CPB}$ with antegrade perfusion, $\%$ (n) & $3.8(8)$ & $100.0(211)$ & $<.001$ \\
\hline Through second ICS, \% (n) & $95.7(202)$ & - & - \\
\hline Through third ICS, \% (n) & $4.3(9)$ & - & - \\
\hline Biological valve, \% (n) & $81.0(171)$ & $73.0(154)$ & .064 \\
\hline Prosthesis size $19, \%(n)$ & $8.5(18)$ & $4.7(10)$ & .170 \\
\hline Prosthesis size $21, \%$ (n) & $34.1(72)$ & $31.3(66)$ & .604 \\
\hline Prosthesis size $23, \%$ (n) & $40.3(85)$ & $43.6(92)$ & .554 \\
\hline Prosthesis size $25, \%$ (n) & $15.6(33)$ & $18.5(39)$ & .518 \\
\hline Prosthesis size $27, \%$ (n) & $1.4(3)$ & $1.0(2)$ & 1.000 \\
\hline Prosthesis size $29, \%$ (n) & $0.0(0)$ & $1.0(2)$ & .499 \\
\hline $\mathrm{Hb}$ concentration (ICU arrival), $\mathrm{g} / \mathrm{dL} \pm \mathrm{SD}$ & $\begin{array}{c}10.3 \pm 3.1 \\
9.5[8.2 ; 13.1]\end{array}$ & $\begin{array}{c}9.8 \pm 3.0 \\
8.9[7.2 ; 11.7]\end{array}$ & .107 \\
\hline $\mathrm{Hb}$ concentration (ICU discharge), $\mathrm{g} / \mathrm{dL} \pm \mathrm{SD}$ & $\begin{array}{c}10.0 \pm 2.7 \\
9.2[8.0 ; 12.0]\end{array}$ & $\begin{array}{c}9.1 \pm 2.7 \\
8.4[6.7 ; 10.8]\end{array}$ & $<.001$ \\
\hline Postoperative drainage during the first $24 \mathrm{~h}, \mathrm{~mL} \pm \mathrm{SD}$ & $\begin{array}{c}353.5 \pm 248.6 \\
260.0[185.0 ; 460.0]\end{array}$ & $\begin{array}{c}544.3 \pm 324.5 \\
500.0[270.0 ; 735.0]\end{array}$ & $<.001$ \\
\hline Patients who required blood transfusion, \% (n) & $48.8(103)$ & $67.3(142)$ & $<.001$ \\
\hline Blood transfused, $\mathrm{mL} \pm \mathrm{SD}$ & $\begin{array}{l}298.1 \pm 317.1 \\
0.0[0.0 ; 540.0]\end{array}$ & $\begin{array}{c}527.7 \pm 427.0 \\
540.0[0.0 ; 810.0]\end{array}$ & $<.001$ \\
\hline 0 units of blood transfused, $\%(n)$ & $51.2(108)$ & $32.7(69)$ & $<.001$ \\
\hline 1 unit of blood transfused, $\%(n)$ & $0.00(0)$ & $0.5(1)$ & 1.000 \\
\hline 2 units of blood transfused, $\%(n)$ & $34.8(77)$ & $27.6(61)$ & .123 \\
\hline 3 units of blood transfused, \% (n) & $11.8(26)$ & $19.9(44)$ & .026 \\
\hline$\geq 4$ units of blood transfused, $\%$ (n) & $0.00(0)$ & $16.3(36)$ & $<.001$ \\
\hline $\mathrm{CPB}$ time, $\min \pm \mathrm{SD}$ & $\begin{array}{c}111.0 \pm 25.0 \\
112.0[90.0 ; 123.0]\end{array}$ & $\begin{array}{c}97.6 \pm 19.4 \\
97.0[82.0 ; 105.0]\end{array}$ & $<.001$ \\
\hline Crossclamp time, $\min \pm \mathrm{SD}$ & $\begin{array}{c}78.2 \pm 13.6 \\
77.0[69.0 ; 80.0]\end{array}$ & $\begin{array}{c}63.4 \pm 12.9 \\
64.0[53.0 ; 72.0]\end{array}$ & $<.001$ \\
\hline Operation time, minutes $\pm \mathrm{SD}$ & $\begin{array}{c}197.1 \pm 27.6 \\
196.0[181.0 ; 211.0]\end{array}$ & $\begin{array}{c}183.0 \pm 51.2 \\
169.0[155.0 ; 180.0]\end{array}$ & $<.001$ \\
\hline Crossclamp time $>90 \min , \%(n)$ & $10.0(21)$ & $2.4(5)$ & $<.001$ \\
\hline CPB time $>120$ min, $\%(n)$ & $34.6(73)$ & $8.1(17)$ & $<.001$ \\
\hline Operation time $>210 \min , \%(\mathrm{n})$ & $27.0(57)$ & $17.1(36)$ & .019 \\
\hline Year $2010(n=44)$ & & & \\
\hline $\mathrm{CPB}$ time, $\min \pm \mathrm{SD}$ & $133.3 \pm 28.2$ & $100.7 \pm 20.6$ & $<.001$ \\
\hline Crossclamp time, $\min \pm \mathrm{SD}$ & $90.9 \pm 20.3$ & $65.4 \pm 14.3$ & $<.001$ \\
\hline Operation time, $\min \pm \mathrm{SD}$ & $221.9 \pm 31.2$ & $183.4 \pm 59.8$ & $<.001$ \\
\hline Year $2011(\mathrm{n}=56)$ & & & \\
\hline $\mathrm{CPB}$ time, $\min \pm \mathrm{SD}$ & $112.2 \pm 23.9$ & $95.8 \pm 18.6$ & $<.001$ \\
\hline Crossclamp time, $\min \pm \mathrm{SD}$ & $78.8 \pm 9.7$ & $62.0 \pm 11.3$ & $<.001$ \\
\hline Operation time, $\min \pm \mathrm{SD}$ & $199.3 \pm 24.9$ & $184.8 \pm 48.4$ & .079 \\
\hline Year $2012(\mathrm{n}=51)$ & & & \\
\hline $\mathrm{CPB}$ time, $\min \pm \mathrm{SD}$ & $102.7 \pm 17.6$ & $98.5 \pm 22.1$ & .277 \\
\hline Crossclamp time, $\min \pm \mathrm{SD}$ & $74.1 \pm 7.5$ & $60.4 \pm 14.2$ & $<.001$ \\
\hline Operation time, $\min \pm \mathrm{SD}$ & $188.2 \pm 21.7$ & $187.5 \pm 53.3$ & .926 \\
\hline Year $2013(n=60)$ & & & \\
\hline $\mathrm{CPB}$ time, $\min \pm \mathrm{SD}$ & $97.4 \pm 18.0$ & $95.4 \pm 19.4$ & .608 \\
\hline Crossclamp time, $\min \pm \mathrm{SD}$ & $70.4 \pm 8.7$ & $61.8 \pm 13.2$ & $<.001$ \\
\hline Operation time, $\min \pm \mathrm{SD}$ & $183.6 \pm 24.1$ & $181.3 \pm 57.9$ & .802 \\
\hline
\end{tabular}

$\mathrm{n}=$ number of patients. Continuous variables are described by mean $\pm \mathrm{SD}$ and by median [first; third quartile]. Categoric variables are shown as percentage. $R T-A V R$, Minimally invasive aortic valve replacement through anterior right minithoracotomy; $A V R$, conventional aortic valve replacment through a median sternotomy; $S D$, standard deviation; $I C U$, intensive care unit; $C P B$, cardiopulmonary bypass; $I C S$, intercostal space; $H b$, hemoglobin. 
TABLE 4. Complications

\begin{tabular}{|c|c|c|c|}
\hline Characteristics & $\begin{array}{c}\text { RT-AVR } \\
\text { group } \\
\mathbf{n}=\mathbf{2 1 1} \\
\end{array}$ & $\begin{array}{c}\text { AVR } \\
\text { group } \\
\mathbf{n}=\mathbf{2 1 1} \\
\end{array}$ & $\begin{array}{c}P \\
\text { value } \\
\end{array}$ \\
\hline Reoperation for bleeding, $\%(\mathrm{n})$ & $3.8(8)$ & $5.2(11)$ & .639 \\
\hline Perioperative myocardial infarction, $\%$ (n) & $1.4(3)$ & $1.9(4)$ & 1.000 \\
\hline Gastrointestinal bleeding, \% (n) & $1.4(3)$ & $1.9(4)$ & 1.000 \\
\hline Stroke, \% (n) & $0.5(1)$ & $1.4(3)$ & .615 \\
\hline Hemodialysis, \% (n) & $0.5(1)$ & $0.5(1)$ & 1.000 \\
\hline Paravalvular leak, \% (n) & $0.9(2)$ & $0.5(1)$ & 1.000 \\
\hline Permanent pacemaker implantation, \% (n) & $1.4(3)$ & $0.0(0)$ & .247 \\
\hline New atrial fibrillation, \% (n) & $12.8(27)$ & $24.6(52)$ & .003 \\
\hline LCOS, $\%(n)$ & $1.0(2)$ & $1.0(2)$ & 1.000 \\
\hline Mediastinitis, \% (n) & $0.0(0)$ & $2.8(6)$ & .040 \\
\hline Superficial chest wound infection, $\%$ (n) & $0.0(0)$ & $1.9(4)$ & .132 \\
\hline Groin wound infection, \% (n) & $2.8(6)$ & $0.0(0)$ & .040 \\
\hline Groin lymphocele, \% (n) & $2.4(5)$ & $0.0(0)$ & .061 \\
\hline Pneumonia, \% (n) & $1.4(3)$ & $0.5(1)$ & .623 \\
\hline Hemothorax, \% (n) & $1.0(2)$ & $0.0(0)$ & .499 \\
\hline Pleural effusion, \% (n) & $6.6(14)$ & $9.0(19)$ & .469 \\
\hline Thoracentesis in hospital, \% (n) & $5.2(11)$ & $7.6(16)$ & .427 \\
\hline
\end{tabular}

decreased from $222.0 \pm 31.2$ minutes in 2010 to $184.0 \pm 24.1$ minutes in $2013(P<.001)$. Postoperative drainage volume diminished over the study period, from $439.0 \pm 214.0 \mathrm{~mL}$ in 2010 to $279.0 \pm 268.0 \mathrm{~mL}$ in 2013 $(P<.001)$. This indicates a learning curve and progress with increased experience (Figure 1).

Multivariable logistic regression analysis showed that the minimally invasive surgical technique applied in the RT-AVR group resulted in reduced postoperative morbidity, analyzed in our model as 1 dependent variable, bleeding, and blood transfusion requirements (Table 5).

Poisson regression analysis revealed that factors that had an influence on the length of ICU stay were the surgical technique, EuroSCORE II, preoperative comorbidities such as renal insufficiency and cerebrovascular disease, amount of blood transfused, and occurrence of postoperative atrial fibrillation. Factors that had an influence on the length of hospital stay were the surgical technique, preoperative comorbidities such as renal insufficiency and cerebrovascular disease, amounts of postoperative drainage and blood transfused, and occurrence of postoperative atrial fibrillation (Table 6).

\section{DISCUSSION}

The main finding of our study is that RT-AVR surgery was performed without increased mortality and morbidity compared with conventional AVR surgery. RT-AVR was feasible and safe, with shorter ICU and hospital stays when compared with standard AVR performed via a median sternotomy.
In our experience, retrograde CPB perfusion did not result in increased stroke rates. The reason may be that we carefully examined patients with MDCT scans of the aorta and femoral arteries, looking for factors increasing the risk of cerebrovascular accidents during retrograde CPB perfusion. ${ }^{14,15}$ The advantages of performing RT-AVR surgery were reductions in postoperative blood loss, amount of blood transfused, infection rate, atrial fibrillation rate, and length of ICU and hospital stays. ${ }^{3,16}$ Reports describing RT-AVR surgery, which allows for the preservation of sternum stability and earlier mobilization of patients in comparison with median sternotomy, are still rare. ${ }^{5,6}$

We observed a high rate of mediastinitis in the conventional AVR group. Moreover, in the RT-AVR group, groin infections or groin lymphocele occurrence was high. More attention should be paid to perfect the surgical technique to avoid these complications. We noticed the problem of lung hernias after the minithoracotomy closure in 4 patients early in our experience. We no longer encounter this problem because we pay careful attention to close the minithoracotomy firmly in layers and fix the costal cartilage to the sternum.

Ligation of the right interior thoracic artery can be a disadvantage of RT-AVR surgery for patients with coronary artery disease who may require future surgical coronary revascularization. During RT-AVR surgery, visualization of the coronary arteries can be impaired. It is crucial to visualize them correctly, safely administer cardioplegia, and protect the coronary orifices from embolization with calcium debris. When this problem occurred, we found thoracoscopic visualization of the aortic valve and root to be helpful.

Minimally invasive aortic valve surgery results in a reduction of pain, lower blood loss, fewer blood transfusions, earlier recovery, and improved patient satisfaction in comparison with the sternotomy approach. ${ }^{3,5,6,17-19}$ It was reported that the atrial fibrillation rate is lower in patients after RT-AVR compared with standard AVR surgery. ${ }^{4,20}$ Others have reported that a reduced rate of new-onset atrial fibrillation after minimally invasive AVR surgery is observed especially in higher-risk elderly patients and that older age is a risk factor for increased occurrence of atrial fibrillation. ${ }^{21}$ It has been speculated that reduced surgical trauma, which can result in reduced postoperative pain, preserved integrity of the thorax, or less impaired respiratory function after minimally invasive surgery can result in a reduced rate of new-onset postoperative atrial fibrillation. ${ }^{22}$ Heart failure is a predictor of postoperative atrial fibrillation. ${ }^{23}$ We did not observe LCOS among patients who experienced postoperative atrial fibrillation. New-onset atrial fibrillation was not observed in patients with LCOS. One potential explanation for this occurrence is that there were few patients who presented LCOS in our material. On the other hand, the frequency of 


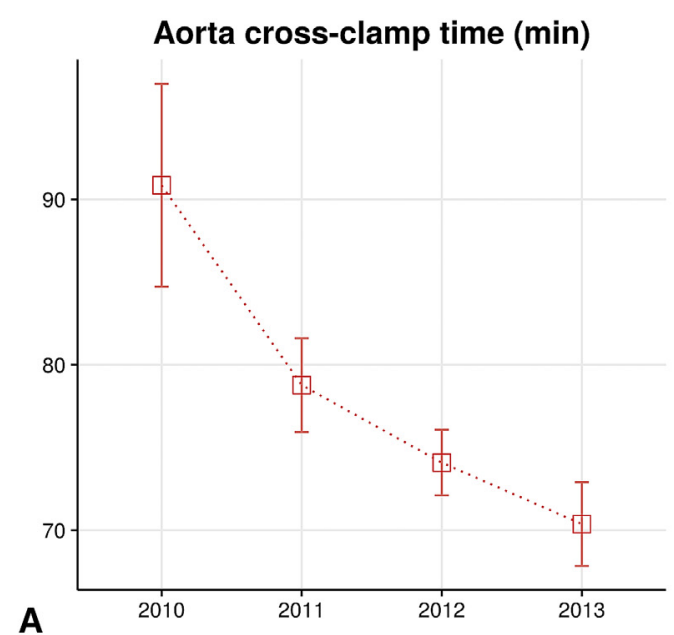

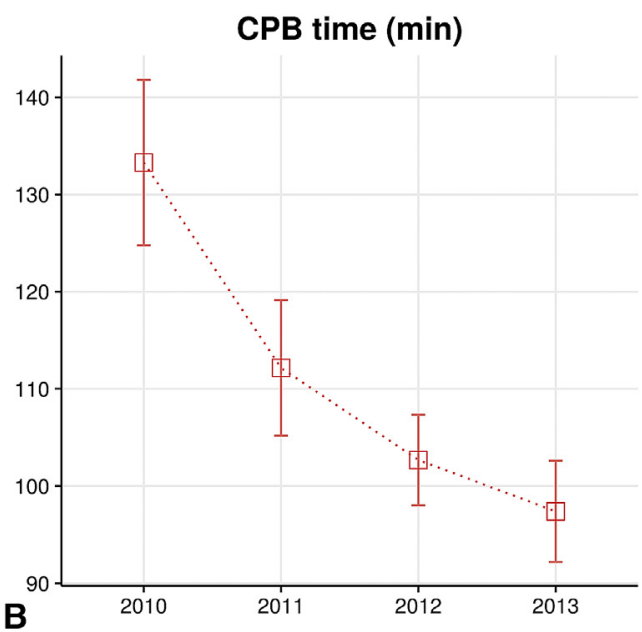

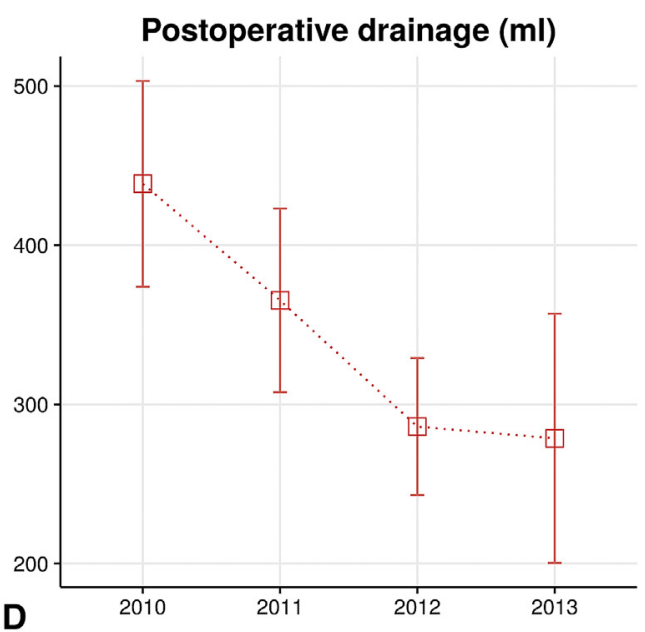

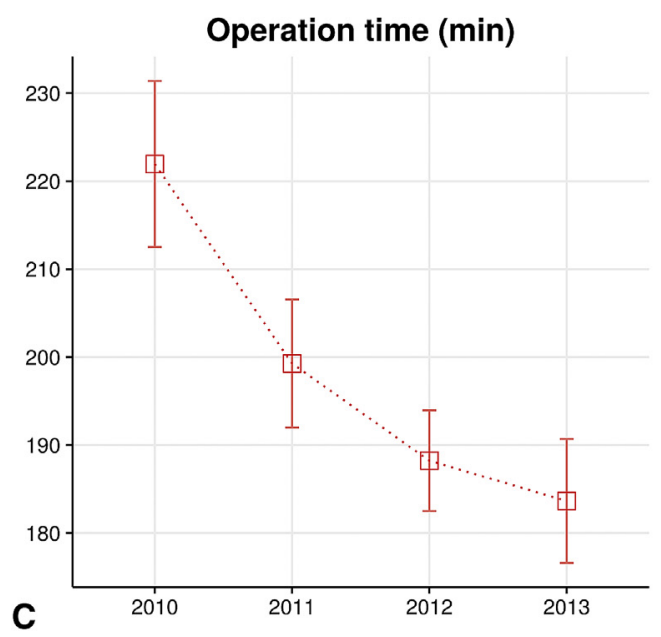

FIGURE 1. Trends over time of aortic crossclamp time (A), CPB time (B), operation time (C), and postoperative drainage (D) in the RT-AVR group. Data are presented as mean values, and confidence limits are included. $2010(\mathrm{n}=44), 2011(\mathrm{n}=56), 2012(\mathrm{n}=51), 2013(\mathrm{n}=60)$. Details are described in the "Results" section. $C P B$, Cardiopulmonary bypass.

new-onset postoperative atrial fibrillation was reduced when RT-AVR surgery was applied.

Multivariable logistic regression analysis demonstrated that applying the RT-AVR technique can reduce the risk of increased postoperative blood loos and blood transfusion requirements. ${ }^{24}$ Perioperative blood loss in our patients was not excessive. Despite this, the hemoglobin level during the ICU stay was 8 to $10 \mathrm{~g} / \mathrm{d}$. One possible explanation is perioperative fluid management both during CPB and after surgery. Fluids were given preferentially rather than inotropic medication to ensure hemodynamic stability and avoid high-pressure gradient in the left ventricle outflow tract in patients with left ventricle hypertrophy. Our patients received blood to prevent low hemoglobin concentration whenever it was indicated.

The surgical technique also appeared as a risk factor of postoperative morbidity. Thus, RT-AVR surgery can reduce the risk of postoperative complications despite the fact that minimally invasive procedures require longer aortic crossclamp, CPB, and operation times. ${ }^{24}$ In our institution we use both warm and cold blood crystalloid cardioplegia. In our experience, no clear clinical benefit has been demonstrated by warm or cold blood cardioplegia, and both provide similar efficacy of myocardial protection as previously reported. ${ }^{25,26}$

Application of sutureless valves for RT-AVR making the surgery shorter could be a minimally invasive surgical alternative to transcatheter techniques, with expected good long-term results. ${ }^{27-29}$ Despite a longer surgery time for RT-AVR, we observed that ICU and hospital stays after RT-AVR surgery were reduced. ${ }^{5,6}$ It is likely that diminished surgical trauma and retained chest wall integrity can result in improved postoperative recovery during the rehabilitation phase and a shorter hospital stay. ${ }^{30}$ The increase in CBP and aorta crossclamp times during RT-AVR surgery is modest and normalizes with the learning curve and increased experience. ${ }^{4,8,20}$ Of note, 
TABLE 5. Multivariable logistic regression analysis of risk factors of postoperative morbidity, bleeding, and blood transfusion requirements

\begin{tabular}{lrrr}
\hline \multicolumn{4}{c}{ Dependent variable } \\
\hline \multicolumn{1}{c}{ Independent variable } & OR & $\mathbf{9 5} \%$ CI & $\boldsymbol{P}$ value \\
\hline Postoperative morbidity & & & \\
$\quad$ (Intercept) & 0.6 & $0.5-0.8$ & .001 \\
RT-AVR group (surgical technique) & 0.5 & $0.3-0.8$ & .001 \\
COPD & 3.7 & $1.7-8.3$ & $<.001$ \\
Cerebrovascular disease & 2.5 & $1.3-5.0$ & .008
\end{tabular}

Postoperative bleeding and blood requirements (postoperative drainage $>800 \mathrm{~mL}$, blood transfusions)

\begin{tabular}{llll} 
(Intercept) & 2.2 & $1.6-3.0$ & $<.001$ \\
RT-AVR group (surgical technique) & 0.4 & $0.3-0.6$ & $<.001$ \\
\hline
\end{tabular}

Input model consisted of the following independent variables: RT-AVR surgical technique, female sex, age, BMI, aortic stenosis, EuroSCORE II, renal insufficiency, diabetes, COPD, EF, hypertension, peripheral vascular disease, coronary artery disease, cerebrovascular disease, New York Heart Association class III or IV, history of myocardial infarction, history of atrial fibrillation, $\mathrm{Hb}$ ( $1 \mathrm{~d}$ before surgery), CPB time, and $\mathrm{Hb}$ (ICU arrival). Final model was obtained by stepwise regression using backward elimination approach in which independent variables with $P$ values greater than .05 are sequentially removed until only statistically significant variables remain. $O R$, Odds ratio; $C I$, confidence interval; $R T-A V R$, minimally invasive aortic valve replacement through a right anterior minithoracotomy; $C O P D$, chronic obstructive pulmonary disease.

in our study of lower-risk patients, the length of ICU or hospital stay correlated with preoperative clinical patient status but also with the surgical technique, postoperative blood drainage, and amount of blood transfused. We did not analyze inflammatory or coagulation markers in the study. However, an additional factor of inflammatory response or changes in coagulation profile could be due to significant surgical stress caused by sternotomy. ${ }^{31}$ Less morbidity after RT-AVR surgery in part could be related to the decreased systemic inflammatory response. ${ }^{32}$ Comparison of inflammatory markers between patients undergoing classic AVR surgery and patients undergoing RT-AVR surgery could help to answer the question of how important a role inflammatory response plays in the range of surgical trauma.

Postoperative atrial fibrillation in cardiac surgery is a known risk factor of prolonged ICU and hospital stays, as well as postoperative complications and increased hospital costs. ${ }^{33}$ In our study, the occurrence of postoperative atrial fibrillation also correlated with ICU and hospital length of stays and resulted in a longer hospitalization period. Minimally invasive RT-AVR surgery with reduced occurrence of postoperative atrial fibrillation, postoperative complications, and shorter length of hospital stay can result in diminished hospitalization costs. ${ }^{33,34}$ Proper preoperative planning with MDCT imaging can minimize the conversion rate resulting from difficult access to the ascending aorta. ${ }^{4,5}$ Most of the conversions occurred early in our experience with RT-AVR surgery. A meticulous surgical technique with perfect hemostasis is crucial to a reduced conversion rate due to bleeding complications. However, pleural or
TABLE 6. Poisson regression analysis for the length of intensive care unit and hospital stays

\begin{tabular}{lrcr}
\hline & \multicolumn{3}{c}{ Length of ICU stay } \\
\cline { 2 - 4 } \multicolumn{1}{c}{ Variable } & Estimate & Standard error & $\boldsymbol{P}$ value \\
\hline (Intercept) & 0.331 & 0.116 & .004 \\
RT-AVR surgery & -0.525 & 0.079 & $<.001$ \\
EuroSCORE II & 0.113 & 0.020 & $<.001$ \\
Renal insufficiency & 1.486 & 0.105 & $<.001$ \\
Cerebrovascular disease & 0.435 & 0.106 & $<.001$ \\
Blood transfused (mL) & 0.000 & 0.000 & $<.001$ \\
Postoperative AF & 0.328 & 0.083 & $<.001$ \\
\hline
\end{tabular}

\begin{tabular}{lrcr} 
& \multicolumn{3}{c}{ Length of hospital stay } \\
\cline { 2 - 4 } \multicolumn{1}{c}{ Variable } & Estimate & Standard error & $\boldsymbol{P}$ value \\
\hline (Intercept) & 1.878 & 0.056 & $<.001$ \\
RT-AVR surgery & -0.292 & 0.041 & $<.001$ \\
Renal insufficiency & 0.189 & 0.058 & .001 \\
Cerebrovascular disease & 0.179 & 0.058 & .002 \\
Postoperative drainage (mL) & 0.000 & 0.000 & .020 \\
Blood transfused (mL) & 0.000 & 0.000 & $<.001$ \\
Postoperative AF & 0.270 & 0.044 & $<.001$ \\
\hline
\end{tabular}

Input model consisted of the following independent variables: RT-AVR surgical technique, female sex, age, BMI, aortic stenosis, EuroSCORE II, renal insufficiency, diabetes, COPD, EF, hypertension, peripheral vascular disease, coronary artery disease, cerebrovascular disease, New York Heart Association class III or IV, history of myocardial infarction, history of atrial fibrillation, $\mathrm{Hb}$ ( $1 \mathrm{~d}$ before surgery), CPB time, $\mathrm{Hb}$ (ICU arrival), postoperative drainage $(\mathrm{mL})$, blood transfused $(\mathrm{mL})$, and postoperative atrial fibrillation. Final model was obtained by stepwise regression using backward elimination approach in which independent variables with $P$ values greater than .05 are sequentially removed until only statistically significant variables remain. ICU, Intensive care unit; $R T-A V R$, minimally invasive aortic valve replacement through a right anterior minithoracotomy; EuroSCORE, European System for Cardiac Operative Risk Evaluation; $A F$, atrial fibrillation.

pericardial adhesions are difficult to predict and remain a significant reason for conversion. ${ }^{35}$

\section{Study Limitations}

This was a single-center study, and only perioperative outcomes were analyzed. Further analysis on posthospital discharge data and long-term outcomes should be performed. This retrospective observational study analyzing prospectively collected data was restricted only to lower-risk patients who underwent AVR surgery.

The study was intrinsically biased, because not every patient was suitable for RT-AVR surgery. The anatomic position of the ascending aorta in relation to the right sternal border is a key factor determining the suitability for this approach. ${ }^{8}$ Randomized trials comparing results of AVR through a median sternotomy with the less-invasive RT-AVR technique should be performed.

Our study lacked assessment of patients' satisfaction, postoperative pain, or costs because these factors were not included in the postoperative protocol and our database. ${ }^{34}$ Finally, it is important to underline that our results refer only to patients after aortic surgery performed through a 
right anterior minithoracotomy and cannot be extrapolated to other minimally invasive techniques.

\section{CONCLUSIONS}

RT-AVR surgery appeared to be feasible, safe, and efficient with excellent cosmetic results and to have a mortality rate comparable to that of conventional AVR surgery. RT-AVR resulted in a reduced infection rate, diminished postoperative bleeding and blood transfusion requirements, reduced occurrence of new-onset atrial fibrillation, and shorter ICU and hospital stays. Potential contraindications for RT-AVR are dense pleural adhesion and the ascending aorta positioned deep under the sternum. On the basis of the good results of RT-AVR compared with conventional AVR, we hypothesize that this surgical technique could offer improved perioperative outcomes for patients with a lower perioperative risk requiring isolated AVR in comparison with conventional AVR surgery.

\section{Conflict of Interest Statement}

Authors have nothing to disclose with regard to commercial support.

\section{References}

1. Hufnagel CA, Vilkgas PD, Nahas H. Experiences with new types of aortic valvular prosthesis. Ann Surg. 1958;147:636-44.

2. Cosgrove DM, Sabik JF. Minimally invasive approach for aortic valve operations. Ann Thorac Surg. 1996;62:596-7.

3. Brown ML, McKellar SH, Sundt TM, Schaff HV. Ministernotomy versus conventional sternotomy for aortic valve replacement: a systematic review and meta-analysis. J Thorac Cardiovasc Surg. 2009;137:670-9.

4. Glauber M, Miceli A, Bevilacqua S, Farneti PA. Minimally invasive aortic valve replacement via right anterior minithoracotomy: early outcomes and midterm follow-up. J Thorac Cardiovasc Surg. 2011;142:1577-9.

5. Lamelas J, Sarria A, Santana O, Pineda AM, Lamas GA. Outcomes of minimally invasive valve surgery versus median sternotomy in patients aged 75 years or greater. Ann Thorac Surg. 2011;91:79-84.

6. von Elm E, Altman DG, Egger M, Pocock SJ, Gøtzsche PC, Vandenbroucke JP. The strengthening the reporting of observational studies in epidemiology (STROBE) statement: guidelines for reporting observational studies. PLoS Med. 2007;4:e296.

7. Brinkman WT, Hoffman W, Dewey TM, Culica D, Prince SL, Herbert MA, et al. Aortic valve replacement surgery: comparison of outcomes in matched sternotomy and PORT ACCESS groups. Ann Thorac Surg. 2010;90:131-5.

8. Glauber M, Ferrarini M, Miceli A. Minimally invasive aortic valve surgery: state of the art and future directions. Ann Cardiothorac Surg. 2015;4:26-32.

9. Gajic O, Malinchoc M, Comfere TB, Harris MR, Achouiti A, Yilmaz M, et al. The Stability and Workload Index for Transfer score predicts unplanned intensive care unit patient readmission: initial development and validation. Crit Care Med. 2008;36:676-82.

10. Nashef SA, Roques F, Sharples LD, Nilsson J, Smith C, Goldstone AR, et al. EuroSCORE II. Eur J Cardiothorac Surg. 2012;41:734-45.

11. Byrne J, Leacche M, Unie D, Rawn J, Simon D, Rogers C, et al. Staged initial percutaneous coronary intervention followed by valve surgery ("hybrid approach") for patients with complex coronary and valve disease. J Am Coll Cardiol. 2005;45:14-8.

12. Goolsby MJ. National Kidney Foundation Guidelines for chronic kidney disease: evaluation, classification, and stratification. J Am Acad Nurse Pract. 2002;14:238-42.

13. $\mathrm{R}$ Development Core Team. $R$ : a language and environment for statistical computing. Vienna, Austria: R Foundation for Statistical Computing; 2010. ISBN 3-900051-07-0. Available at: http://www.R-project.org. Accessed October $1,2015$.
14. Lamelas J, Nguyen TC. Minimally invasive valve surgery: when less is more Semin Thorac Cardiovasc Surg. 2015;27:49-56.

15. Goldstone AB, Joseph Woo Y. Minimally invasive surgical treatment of valvular heart disease. Semin Thorac Cardiovasc Surg. 2014;26:36-43.

16. Bakir I, Casselman FP, Wellens F, Jeanmart H, De Geest R, Degrieck I, et al. Minimally invasive versus standard approach aortic valve replacement: a study in 506 patients. Ann Thorac Surg. 2006;81:1599-604.

17. Merk D, Lehmann S, Holzhey D, Dohmen P, Candolfi P, Misfeld M, et al. Minimal invasive aortic valve replacement surgery is associated with improved survival: a propensity-matched comparison. Eur J Cardiothorac Surg. 2015;47:11-7.

18. Phan K, Xie A, Tsai YC, Black D, Di Eusanio M, Yan TD. Ministernotomy or minithoracotomy for minimally invasive aortic valve replacement: a Bayesiar network meta-analysis. Ann Cardiothorac Surg. 2015;4:3-14.

19. Tabata M, Umakanthan R, Cohn LH, Bolman RM, Shekar PS, Chen FY, et al. Early and late outcomes of 1000 minimally invasive aortic valve operations. Eur J Cardiothorac Surg. 2008;33:537-41.

20. Gilmanov D, Bevilacqua S, Murzi M, Cerillo AG, Gasbarri T, Kallushi E, et al. Minimally invasive and conventional aortic valve replacement: a propensity score analysis. Ann Thorac Surg. 2013;96:837-43.

21. De Smet JM, Rondelet B, Jansens JL, Antoine M, De Canniere D, Le Clerc JL. Assessment based on EuroSCORE of ministernotomy for aortic valve replacement. Asian Cardiovasc Thorac Ann. 2004;12:53-7.

22. Glauber M, Miceli A, Gilmanov D, Ferrarini M, Bevilacqua S, Farneti P, et al. Right anterior minithoracotomy versus conventional aortic valve replacement: a propensity score matched study. J Thorac Cardiovasc Surg. 2013;145:1222-6.

23. Banach M, Goch A, Misztal M, Rysz J, Jaszewski R, Goch JH. Predictors of paroxysmal atrial fibrillation in patients undergoing aortic valve replacement. J Thorac Cardiovasc Surg. 2007:134:1569-76.

24. Bowdish ME, Hui DS, Cleveland JD, Mack WJ, Sinha R, Ranjan R, et al A comparison of aortic valve replacement via an anterior right minithoracotomy with standard sternotomy: a propensity score analysis of 492 patients. Eur $J$ Cardiothorac Surg. 2016;49:456-63.

25. Abah U, Garfjeld Roberts P, Ishaq M, De Silva R. Is cold or warm blood cardioplegia superior for myocardial protection? Interact Cardiovasc Thorac Surg. 2012;14:848-55.

26. Fan Y, Zhang AM, Xiao YB, Weng YG, Hetzer R. Warm versus cold cardioplegia for heart surgery: a meta-analysis. Eur J Cardiothorac Surg. 2010;37:912-9.

27. Miceli A, Gilmanov D, Murzi M, Marchi F, Ferrarini M, Cerillo AG, et al Minimally invasive aortic valve replacement with a sutureless valve through a right anterior mini-thoracotomy versus transcatheter aortic valve implantation in high-risk patients. Eur J Cardiothorac Surg. 2016;49:960-5.

28. Englberger L, Carrel TP, Doss M, Sadowski J, Bartus K, Eckstein FF, et al. Clinical performance of a sutureless aortic bioprosthesis: five-year results of the $3 \mathrm{f}$ Enable long-term follow-up study. J Thorac Cardiovasc Surg. 2014;148:1681-7.

29. Glauber M, Ferrarini M, Miceli A. Rapid deployment aortic valves and minimally invasive aortic valve replacement: a perfect marriage of technology and technique? J Thorac Cardiovasc Surg. 2015;149:441-2.

30. Bonacchi M, Prifti E, Giunti G, Salica A, Frati G, Sani G. Respiratory dysfunction after coronary artery bypass grafting employing bilateral internal mammary arteries: the influence of intact pleura. Eur J Cardiothorac Surg. 2001;19:827-33

31. Suleiman MS, Zacharowski K, Angelini GD. Inflammatory response and cardioprotection during open-heart surgery: the importance of anaesthetics. Br J Pharmacol. 2008;153:21-33.

32. Karu I, Taal G, Zilmer K, Pruunsild C, Starkopf J, Zilmer M. Inflammatory/ oxidative stress during the first week after different types of cardiac surgery. Scand Cardiovasc J. 2010;44:119-24.

33. Kaireviciute D, Aidietis A, Lip G. Atrial fibrillation following cardiac surgery: clinical features and preventative strategies. Eur Heart J. 2009;30:410-25.

34. Ghanta RK, Lapar DJ, Kern JA, Kron IL, Speir AM, Fonner E Jr, et al. Minimally invasive aortic valve replacement provides equivalent outcomes at reduced cost compared with conventional aortic valve replacement: a realworld multi-institutional analysis. J Thorac Cardiovase Surg. 2015;149:1060-5.

35. Miceli A, Murzi M, Gilmanov D, Fugà R, Ferrarini M, Solinas M, et al Minimally invasive aortic valve replacement using right minithoracotomy is associated with better outcomes than ministernotomy. $J$ Thorac Cardiovasc Surg. 2014;148:133-7.

Key Words: aortic valve replacement, minimally invasive aortic valve surgery, right anterior minithoracotomy 